\title{
Early goal-directed and lactate-guided therapy in adult patients with severe sepsis and septic shock: a meta-analysis of randomized controlled trials
}

Xian-Fei Ding ${ }^{1,2+}$, Zi-Yue Yang $^{1 \dagger}$, Zhen-Tao Xu ${ }^{1,2+}$, Li-Feng Li ${ }^{3,4}$, Bo Yuan ${ }^{1,2}$, Li-Na Guo $^{5}$, Le-Xin Wang ${ }^{6}$, Xi Zhu ${ }^{7^{*}}$ and Tong-Wen Sun ${ }^{1,2^{*}}$

\begin{abstract}
Background: The ProCESS, ARISE, and ProMISe trials have failed to show that early goal-directed therapy (EGDT) reduces mortality in patients with severe sepsis and septic shock. Although lactate-guided therapy (LGT) has been shown to result in significantly lower mortality, its use remains controversial. Therefore, we performed a meta-analysis to evaluate EGDT vs. LGT or usual care (UC) in adult patients with severe sepsis and septic shock.

Methods: Relevant randomized controlled trials published from January 1, 2001 to March 30, 2017 were identified in PubMed, EMBASE, Web of Science, and the Cochrane Library. The primary outcome was mortality; secondary outcomes included red cell transfusions, dobutamine use, vasopressor infusion, and mechanical ventilation support within the first $6 \mathrm{~h}$ and Acute Physiology and Chronic Health Evaluation II (APACHE II) score.

Results: Sixteen studies enrolling 5968 patients with 2956 in EGDT, 2547 in UC, and 465 in LGT were included in this meta-analysis. Compared with UC, EGDT was associated with a lower mortality ( 10 trials; RR $0.85,95 \% \mathrm{Cl} 0.74-0.97$, $P=0.01$ ), and this difference was more pronounced in the subgroup of UC patients with mortality $>30 \%$. In addition, EGDT patients received more red cell transfusions, dobutamine, and vasopressor infusions within the first $6 \mathrm{~h}$. Compared with LGT, EGDT was associated with higher mortality (6 trials; RR $1.42,95 \% \mathrm{Cl} 1.19-1.70, P=0.0001$ ) with no heterogeneity $\left(P=0.727, P^{2}=0 \%\right)$.

Conclusion: EGDT seems to reduce mortality in adult patients with severe sepsis and septic shock, and the benefit may primarily be attributed to red cell transfusions, dobutamine administration, and vasopressor infusions within the first $6 \mathrm{~h}$. However, LGT may result in a greater mortality benefit than EGDT.
\end{abstract}

Keywords: EGDT, Lactate-guided therapy, Usual care, Sepsis, Meta-analysis

\footnotetext{
*Correspondence: xizhuccm@163.com; suntongwen@163.com

${ }^{+}$Xian-Fei Ding, Zi-Yue Yang and Zhen-Tao Xu contributed equally to this

work

${ }^{1}$ General ICU, The First Affiliated Hospital of Zhengzhou University,

Zhengzhou 450052, China ${ }^{7}$ Department of Critical Care Medicine, Peking

University Third Hospital, Beijing 100191, China

Full list of author information is available at the end of the article
}

(c) The Author(s) 2018. This article is distributed under the terms of the Creative Commons Attribution 4.0 International License (http://creativecommons.org/licenses/by/4.0/), which permits unrestricted use, distribution, and reproduction in any medium, provided you give appropriate credit to the original author(s) and the source, provide a link to the Creative Commons license, and indicate if changes were made. The Creative Commons Public Domain Dedication waiver (http://creativecommons.org/ publicdomain/zero/1.0/) applies to the data made available in this article, unless otherwise stated. 


\section{Background}

Sepsis is a physiological, pathological, and biochemical syndrome induced by infection and is a major public health concern, accounting for more than $\$ 20$ billion (5.2\%) of total US hospital costs in 2011 [1]. Although its true incidence is unknown, conservative estimates indicate that sepsis is a leading cause of mortality and critical illness worldwide [2, 3].

In 2001, Rivers et al. [4] conducted a 263-patient, single-centre RCT comparing early goal-directed therapy (EGDT) with usual care (UC) in patients with septic shock visiting an urban emergency department (ED) in the US. EGDT was provided through a 6 -h resuscitation protocol that involved the administration of intravenous fluids, vasopressors, inotropes, and red cell transfusions to achieve pre-specified targets for arterial blood pressure, central venous pressure, systemic central venous oxygen saturation $\left(\mathrm{ScVO}_{2}\right)$, and haemoglobin level. EGDT was found to reduce hospital mortality from 46.5 to $30.5 \%$ [4], prompting its adoption by many institutions worldwide [5]. Subsequently, three large multicentre RCTs, namely, the ProCESS [6], ARISE [7], and ProMISe [8] trials, failed to show that EGDT reduced mortality to a greater extent than UC in patients with severe sepsis and septic shock; however, no harm was associated with the intervention strategies. Of note, the patients in these trials [6-8] were less severely ill (lower baseline lactate levels, $\mathrm{Scvo}_{2}$ at or above the target value on admission, and lower mortality in the control group). Although EGDT cannot currently be recommended with the available evidence base, bedside clinicians still need guidance regarding how to approach this group of patients, who have significant mortality and morbidity [9], and the use of EGDT is still safe and may be considered.

Lactate is a standard laboratory parameter measured with prescribed techniques and may serve as a more objective surrogate for tissue perfusion than physical examination or urine output. Additionally, we know that increased lactate levels are associated with worse outcomes [10]. Lactate clearance, defined by the change in lactate levels between 2 points in time, is a much more rapid, less invasive and less costly measurement than physical examination [11, 12]. Lactate clearance and $\mathrm{Scvo}_{2}$ can be used as markers of early recovery of fluid targets, as the lactate clearance rate has been found to be an accurate and reliable indicator of fluid status [13]. In 2004, lactate clearance was shown to be comparable to $\mathrm{Scvo}_{2}$ as a perfusion marker and may therefore function as an instructional target in early resuscitation for septic shock [11]. Accordingly, 6 RCTs have shown significantly lower mortality with LGT resuscitation versus other protocols [13-18]. In addition, several studies have suggested that a significant degree of technical difficulty is associated with the use of computerized spectrophotometric catheters to simultaneously monitor $\mathrm{Scvo}_{2}$. Using $\mathrm{Scvo}_{2}$ requires preplanned training and real-time calibration, and it may be limited by time, expertise, and the need for specialized equipment [19]. Therefore, the prospect of monitoring lactate clearance has gradually attracted interest, and the Surviving Sepsis Campaign (SSC) 2016 also suggested guiding resuscitation by normalizing lactate in patients with elevated lactate levels as a marker of tissue hypoperfusion [9]. However, the use of LGT to guide resuscitation remains controversial.

Previous meta-analyses [20-23] of EGDT vs. UC did not assess studies with interventions that included red cell transfusion, dobutamine use, vasopressor infusion, and mechanical ventilation support within the first $6 \mathrm{~h}$ and Acute Physiology and Chronic Health Evaluation II (APACHE II) scores. Therefore, we conducted this metaanalysis to evaluate the effect of EGDT compared with LGT or UC on mortality among adults with severe sepsis and septic shock.

\section{Methods}

We conducted a meta-analysis using the guidelines recommended by the Cochrane Collaboration in the Cochrane Handbook for Systematic Reviews of Interventions (http://www.cochranehandbook.org). Our meta-analysis was conducted and reported based on the Preferred Reporting Items for Systematic Reviews and Meta-Analyses (PRISMA) guidelines [24]. The PRISMA 2009 checklist is shown in Additional file 1.

\section{Search strategy}

The PubMed, EMBASE, ISI Web of Science, and Cochrane Library databases were searched systematically to identify all RCTs published from January 1, 2001, to March 30, 2017, assessing EGDT in adult patients with severe sepsis and septic shock. The search process is shown in Fig. 1. The following terms were used: "Early goal-directed therapy" or "EGDT", "lactate", and "sepsis" or "septic shock" or "severe sepsis". There were no language restrictions.

\section{Study selection}

Two authors independently checked the title and abstract of the studies to determine whether they potentially met the inclusion criteria. We also recorded the reasons for excluding trials. We resolved disagreements through discussion. 
PRISMA 2009 Flow Diagram

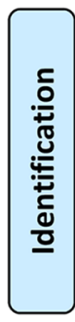

1239 potentially relevant articles

403 from Medine/Pubmed

774 from Embase/OVID

62 from Cochrane library

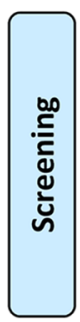

405 articles screened based on titles and abstracts

834 duplicated

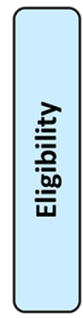

18 articles screened based

387 excluded

70 meta-analyses

12 animal studies

250 not adult

13 reviews

42 not RCT on full-text

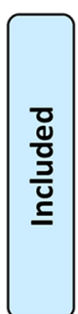

2 excluded

1 not EGDT

1 no data on outcome of interests

Fig. 1 Flow chart for the study selection

Inclusion criteria

\section{Patients}

Relevant RCT studies compared EGDT with LGT or UC in adults with severe sepsis and septic shock.

\section{Interventions}

Standard EGDT was defined as outlined in the SSC, namely, achieving central venous pressure of 8 to $12 \mathrm{mmHg}$, mean arterial pressure of 65 to $90 \mathrm{mmHg}$, urine output of $0.5 \mathrm{ml} / \mathrm{kg} / \mathrm{h}$ or more, and $\mathrm{Scvo}_{2}$ of $70 \%$ or above within the first $6 \mathrm{~h}$ of intervention [25].

\section{Comparison}

Control care, including UC (UC was defined as conventional treatment, which was at the discretion of the 
clinicians) and LGT (lactate or lactate clearance monitoring with the aim to decrease levels), was used for comparison.

\section{Outcomes}

The primary endpoint was all-cause mortality. When several mortality rates were reported in one study, we used the primary outcome. Secondary outcomes included red cell transfusion, dobutamine use, vasopressor infusion, mechanical ventilation support within the first $6 \mathrm{~h}$, and APACHE II scores.

\section{Study design}

We included all RCTs comparing EGDT with UC or LGT in patients with severe sepsis or septic shock.

\section{Exclusion criteria}

The exclusion criteria were as follows: (1) studies with duplicated data; (2) non-randomized studies; (3) metaanalyses, animal studies, studies of non-adult populations, or reviews; and (4) studies published before January 2001.

\section{Data extraction}

Two authors independently extracted the data from each trial, including the name of the first author, year of publication, demographic characteristics (population, country, clinical setting), centre, number of patients, mortality, study design, and APACHE II score.

\section{Risk of bias}

Reviewers independently assessed the risk of bias using the Cochrane collaboration tool [24]. Two authors respectively rated the risks (as "low", "unclear" or "high" risk of bias) regarding 7 domains: random sequence generation, allocation concealment, blinding of participants and personnel, blinding of outcome assessment, incomplete outcome data, selective reporting, and other bias. High risk of bias in any one or more of the key domains of research indicated that the study had a high risk of bias. Studies with a low risk of bias in all key domains were considered to have a low risk of bias overall. All other studies were considered to have an unclear risk of bias.

\section{Statistical analysis}

For dichotomous data, we estimated the relative risk (RR) and 95\% confidence interval (CI) to describe the size of the treatment effect. We performed all analyses using the intention-to-treat principle and generated funnel plots to evaluate publication bias. We quantified statistical heterogeneity using the $I^{2}$ statistic, calculated as $I^{2}=100 \% \times(\mathrm{Q}-d f) / \mathrm{Q}$, where $\mathrm{Q}$ is Cochran's heterogeneity statistic [26]. $I^{2}$ values of $0-24.9 \%$ indicated no heterogeneity, $25-49.9 \%$ indicated mild heterogeneity, $50-74.9 \%$ indicated moderate heterogeneity, and $75-100 \%$ indicated considerable heterogeneity. The pooled RR of each study was calculated using the fixedeffects model (Mantel-Haenszel) or random-effects model (DerSimonian and Laird). All reported $P$ values were two-sided, and $P$ values $<0.05$ were considered to indicate statistically significant differences. All analyses were performed using Review Manager Software (RevMan, version 5.3).

\section{Results}

\section{Literature search}

The PRISMA statement flowchart shows the literature screening process, study selection, and reasons for exclusion, as presented in Fig. 1. The literature search yielded 1239 potentially relevant articles. We removed 834 duplicate studies based on title screening, and after the abstract evaluation, 387 studies were excluded for not meeting the inclusion criteria. After the full texts were reviewed, a total of 16 RCTs [4, 6-8, 13-18, 27-32] $(\mathrm{n}=5968)$ were finally included in this meta-analysis. There was $100 \%$ agreement between the 2 reviewers regarding study inclusion and exclusion.

\section{Study characteristics}

The main characteristics extracted from the 16 RCTs $[4,6-8,13-18,27-32]$ are shown in Table 1 . This metaanalysis enrolled 5968 patients:2956 in the EGDT, 2547 in the UC, and 465 in the LGT groups. These trials were published from January 1, 2001, to March 30, 2017, with sample sizes ranging from 33 to 1588. Of the 16 trials, 6 $[6,7,14-16,27]$ were multi-centre studies, and the others $[4,8,13,17,18,24-26,28-32]$ were single-centre studies. Ten trials $[4,6-8,27-32]$ reported EGDT vs. UC, and 6 trials [13-18] reported EGDT vs. LGT. There were 3 trials $[4,6,15]$ from the USA, 1 trial [32] from Taiwan, 1 trial [28] from Zambia, 1 trial [7] from Australasia/New Zealand, 1 trial [8] from England, 1 trial [14] from the Netherlands, and 8 trials $[13,16-18,27,29,30,32]$ from China. Ten trials $[13,14,16-18,27,29-32]$ were conducted in the intensive care unit (ICU), 4 trials $[4,7,15$, $16]$ were conducted in the ED, and the remaining trials were conducted in the ICU or ED.

\section{Risk of bias and quality of evidence}

The assessment of risk of bias is shown in Fig. 2. Nine trials $[4,6-8,14-16,28,31]$ were considered to have a low risk of bias, and the remaining 7 trials $[17,18,25,27,29$, 


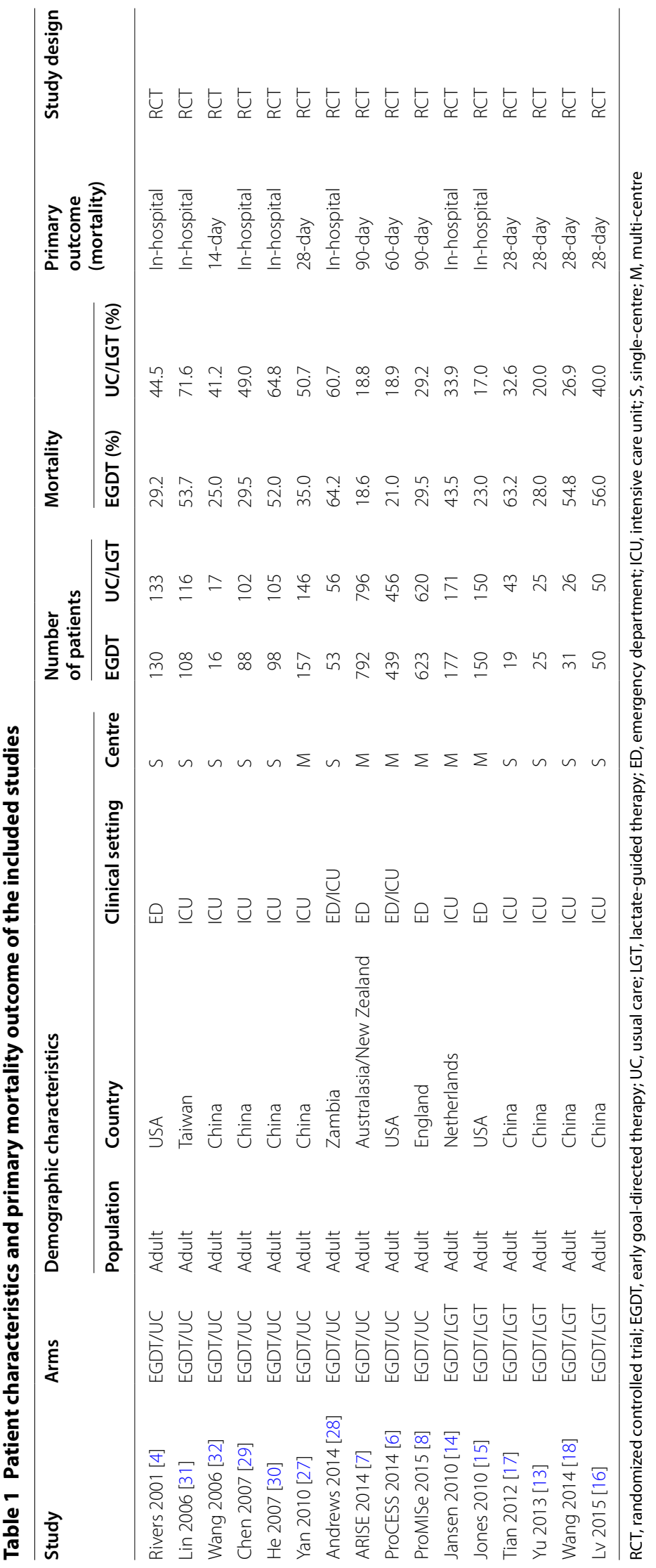




\begin{tabular}{|c|c|c|c|c|c|c|c|c|c|c|c|c|c|c|c|c|}
\hline $\begin{array}{l}\cong \\
\stackrel{\widetilde{G}}{\omega}\end{array}$ & 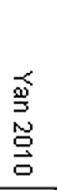 & 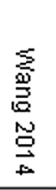 & 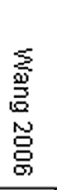 & $\begin{array}{l}\stackrel{-1}{\stackrel{3}{3}} \\
\stackrel{N}{\Xi} \\
\stackrel{N}{N}\end{array}$ & 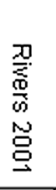 & 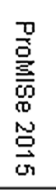 & 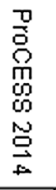 & \begin{tabular}{l}
\multicolumn{2}{c}{} \\
$\stackrel{0}{G}$ \\
$\vec{G}$
\end{tabular} & 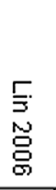 & 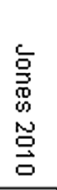 & 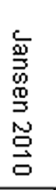 & 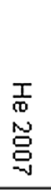 & 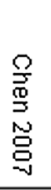 & 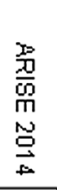 & 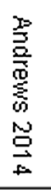 & \\
\hline$\odot$ & $\odot$ & + & $\cdot v$ & + & $\odot$ & $\odot$ & $\odot$ & $\odot$ & 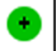 & $\odot$ & $\odot$ & $\sim$ & $\cdot$ & 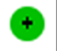 & 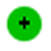 & Random sequence generation (selection bias) \\
\hline$\sim$ & $\cdot \sim$ & + & $\cdot \nu$ & $\cdot \nu$ & + & $\odot$ & $\odot$ & $\odot$ & $\odot$ & $\odot$ & $\odot$ & $\sim$ & $\cdot v$ & 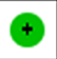 & $\odot$ & Allocation concealment (selection bias) \\
\hline$\odot$ & $\odot$ & $\odot$ & $\odot$ & $\odot$ & $\odot$ & $\odot$ & $\odot$ & $\odot$ & + & 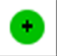 & + & $\odot$ & + & $\odot$ & $\odot$ & Blinding of participants and personnel (performance bias) \\
\hline$\odot$ & $\odot$ & $\odot$ & $\odot$ & + & $\odot$ & $\odot$ & $\odot$ & $\odot$ & $\odot$ & $\odot$ & $\odot$ & $\odot$ & $\odot$ & + & $\odot$ & Blinding of outcome assessment (detection bias) \\
\hline$\odot$ & $\odot$ & $\cdot v$ & + & $\odot$ & + & + & $\odot$ & $\odot$ & + & $\odot$ & + & $\odot$ & + & + & + & Incomplete outcome data (attrition bias) \\
\hline$\odot$ & $\odot$ & $\cdot v$ & + & + & + & $\odot$ & $\odot$ & $\odot$ & + & 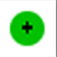 & + & $\sim$ & + & + & + & Selective reporting (reporting bias) \\
\hline$\odot$ & $\odot$ & $\odot$ & $\odot$ & $\odot$ & $\odot$ & $\odot$ & $\odot$ & $\odot$ & + & $\odot$ & $\odot$ & $\odot$ & $\sim$ & + & $\odot$ & Other bias \\
\hline
\end{tabular}

Fig. 2 Risk of bias summary reviewing authors' evaluations of each risk of bias item for each included study. Green circles indicate low risk of bias, yellow circles indicate unclear risk of bias, and red circles indicate high risk of bias

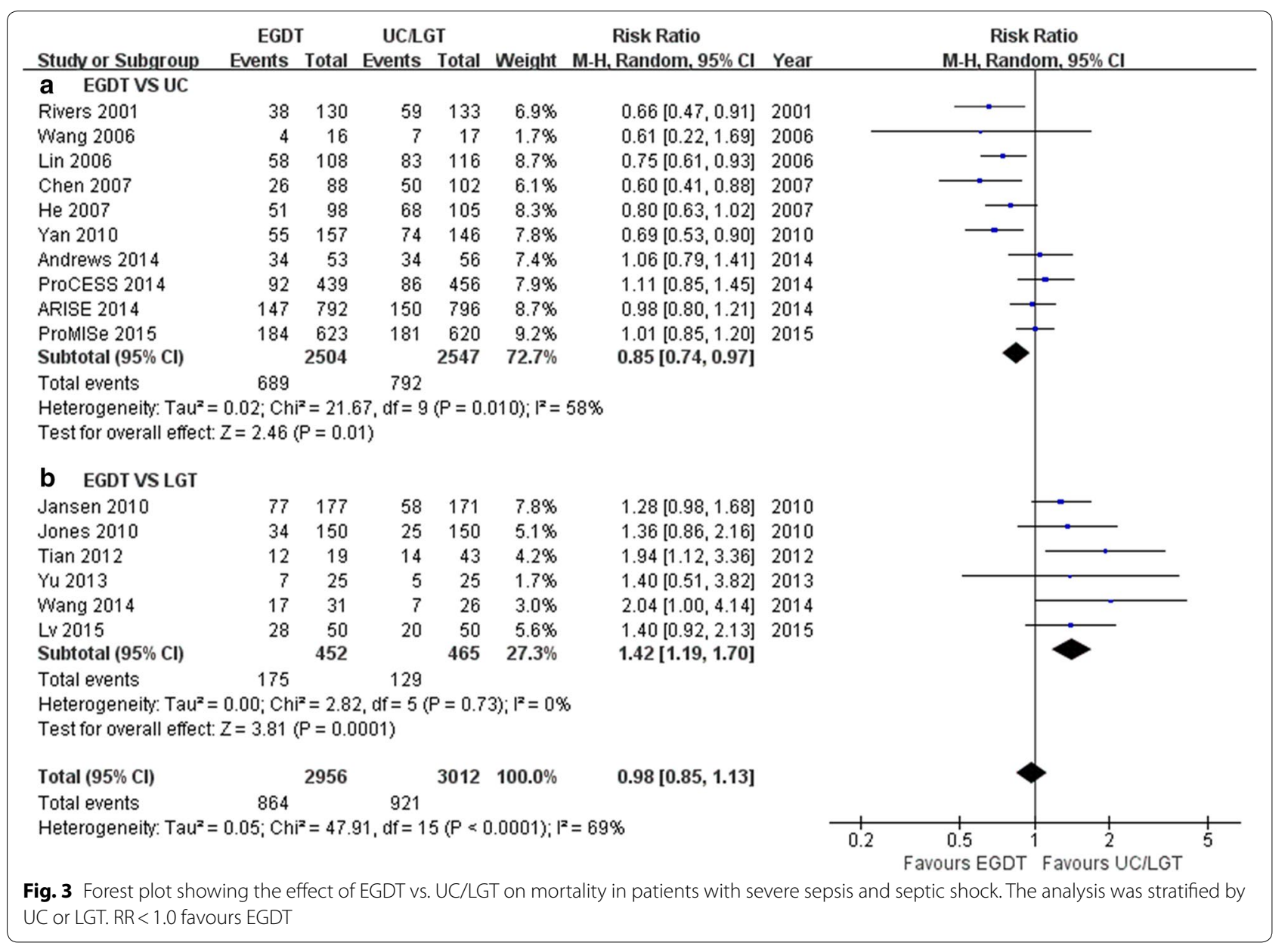


30, 32] had an unclear risk of bias. All trials were RCTs. However, blinding of patients and clinicians to evaluate a complex intervention such as EGDT is extremely difficult, and the authors concluded that the primary outcome was unlikely to be influenced by the lack of blinding.

\section{Primary outcome}

Due to the moderate heterogeneity $\left(I^{2}=69 \%, P<0.0001\right)$ of the pooled RR from 16 trials, a random-effects model was used for the meta-analysis of EGDT versus (UC+LGT). Ten trials [4, 6-8, 27-32] containing 5051 patients compared EGDT with UC in terms of allcause mortality. Compared with UC, EGDT was associated with lower mortality (RR 0.85 , 95\% CI 0.74-0.97, $P=0.01 ; I^{2}=58.5 \%, P=0.010$; Fig. 3). Six trials [13-18] including 917 patients compared the mortality of EGDT with that of LGT. Compared with LGT, EGDT was associated with higher mortality, with no heterogeneity (RR $1.42,95 \%$ CI $1.19-1.70, P=0.0001 ; I^{2}=0 \%, P=0.73$; Fig. 3).

Subgroup analyses according to publication year showed that the mortality benefit of EGDT was observed only in the subgroup of RCTs published prior to the SSC2012 (6 trials; $\mathrm{RR}=0.71,95 \%$ CI $0.63-0.80$, $\left.P<0.0001 ; I^{2}=0 \%, P=0.79\right)[4,27,29-32]$ and not in the subgroup of RCTs published after the SSC 2012 by the fixed-effects model ( 4 trials; $R R=1.02$, 95\% CI 0.92-1.15, $\left.P=0.67 ; I^{2}=0 \%, P=0.90\right)[6-8,28]$ (Fig. 4).

Further subgroup analyses revealed that EGDT had a lower mortality than UC when the mortality of $\mathrm{UC}$ was $>30 \%$ (7 trials; $\mathrm{RR}=0.74,95 \%$ CI $0.66-0.83$, $\left.P<0.0001 ; I^{2}=27 \%, P=0.22\right)[4,27-32]$ but not when the mortality of UC was $<30 \%$ ( 3 trials; $R R=1.02,95 \%$ CI $\left.0.91-1.15, P=0.72 ; I^{2}=0 \%, P=0.77\right)$ [6-8]. In addition, EGDT resulted in higher mortality than LGT when the mortality of the LGT group was both $>$ and $<30 \%$ (3 trials, $\mathrm{RR}=1.37,95 \%$ CI $1.11-1.69, P=0.003 ; I^{2}=0 \%$, $P=0.41[14,16,17]$ and 3 trials, $R R=1.50,95 \%$ CI $1.05-$ $2.16, P=0.003 ; I^{2}=0 \%, P=0.64[13,15,18]$, respectively) (Fig. 5). Due to the low heterogeneity in the subgroup analyses of the mortality, the fixed-effects model was used for the meta-analysis.

\section{Secondary outcomes}

The details of the interventions used in the included studies $[4,6-8,13-18,27-32]$ are shown in Table 2 . These

\begin{tabular}{|c|c|c|c|c|c|c|c|c|c|c|}
\hline Studv or Subgroup & \multicolumn{2}{|c|}{ EGDT } & \multicolumn{2}{|c|}{ UC } & Weight & $\begin{array}{l}\text { Risk Ratio } \\
\text { M-H, Fixed, } 95 \% \mathrm{Cl}\end{array}$ & \multicolumn{4}{|c|}{$\begin{array}{c}\text { Risk Ratio } \\
\text { M-H, Fixed. } 95 \% \mathrm{Cl} \\
\end{array}$} \\
\hline \multicolumn{11}{|c|}{ a $\quad$ EGDT versus UC prior to SSC 2012} \\
\hline Chen 2007 & 26 & 88 & 50 & 102 & $5.9 \%$ & $0.60[0.41,0.88]$ & & $\rightarrow-$ & & \\
\hline He 2007 & 51 & 98 & 68 & 105 & $8.4 \%$ & $0.80[0.63,1.02]$ & & $\rightarrow$ & & \\
\hline $\operatorname{Lin} 2006$ & 58 & 108 & 83 & 116 & $10.2 \%$ & $0.75[0.61,0.93]$ & & $\rightarrow$ & & \\
\hline Rivers 2001 & 38 & 130 & 59 & 133 & $7.5 \%$ & $0.66[0.47,0.91]$ & & $\rightarrow$ & & \\
\hline Wang 2006 & 4 & 16 & 7 & 17 & $0.9 \%$ & $0.61[0.22,1.69]$ & & 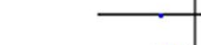 & & \\
\hline Yan 2010 & 55 & 157 & 74 & 146 & $9.8 \%$ & $0.69[0.53,0.90]$ & & - & & \\
\hline Subtotal $(95 \% \mathrm{Cl})$ & & 597 & & 619 & $42.7 \%$ & $0.71[0.63,0.80]$ & & $\downarrow$ & & \\
\hline Total events & 232 & & 341 & & & & & & & \\
\hline \multicolumn{11}{|c|}{$\begin{array}{l}\text { Heterogeneity: } C h i^{2}=2.40, d f=5(P=0.79) ; I^{2}=0 \% \\
\text { Test for overall effect: } Z=5.61(P=0.00001)\end{array}$} \\
\hline \multicolumn{11}{|c|}{ b EGDT versus UC after SSC 2012} \\
\hline Andrews 2014 & 34 & 53 & 34 & 56 & $4.2 \%$ & $1.06[0.79,1.41]$ & & & - & \\
\hline ARISE 2014 & 147 & 792 & 150 & 796 & $19.1 \%$ & $0.98[0.80,1.21]$ & & & & \\
\hline ProCESS 2014 & 92 & 439 & 86 & 456 & $10.8 \%$ & $1.11[0.85,1.45]$ & & & - & \\
\hline PromISe 2015 & 184 & 623 & 181 & 620 & $23.2 \%$ & $1.01[0.85,1.20]$ & & & 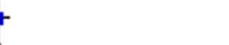 & \\
\hline Subtotal $(95 \% \mathrm{Cl})$ & & 1907 & & 1928 & $57.3 \%$ & $1.02[0.92,1.15]$ & & & & \\
\hline Total events & 457 & & 451 & & & & & & & \\
\hline \multicolumn{11}{|c|}{$\begin{array}{l}\text { Heterogeneity: } \mathrm{Chi}^{2}=0.57, \mathrm{df}=3(\mathrm{P}=0.90) ; \mathrm{I}^{2}=0 \% \\
\text { Test for overall effect: } Z=0.43(P=0.67)\end{array}$} \\
\hline Total $(95 \% \mathrm{Cl})$ & & 2504 & & 2547 & $100.0 \%$ & $0.89[0.82,0.97]$ & & 1 & & \\
\hline Total events & 689 & & 792 & & & & & & & \\
\hline \multicolumn{7}{|c|}{ Heterogeneity: $\mathrm{Chi}^{2}=21.67, \mathrm{df}=9(P=0.010) ; \mathrm{I}^{2}=58 \%$} & 0.01 & $\begin{array}{cc}0.1 & 1 \\
\text { Favours EGDT }\end{array}$ & $\begin{array}{c}10 \\
\text { Favours UC/LGT }\end{array}$ & 100 \\
\hline
\end{tabular}




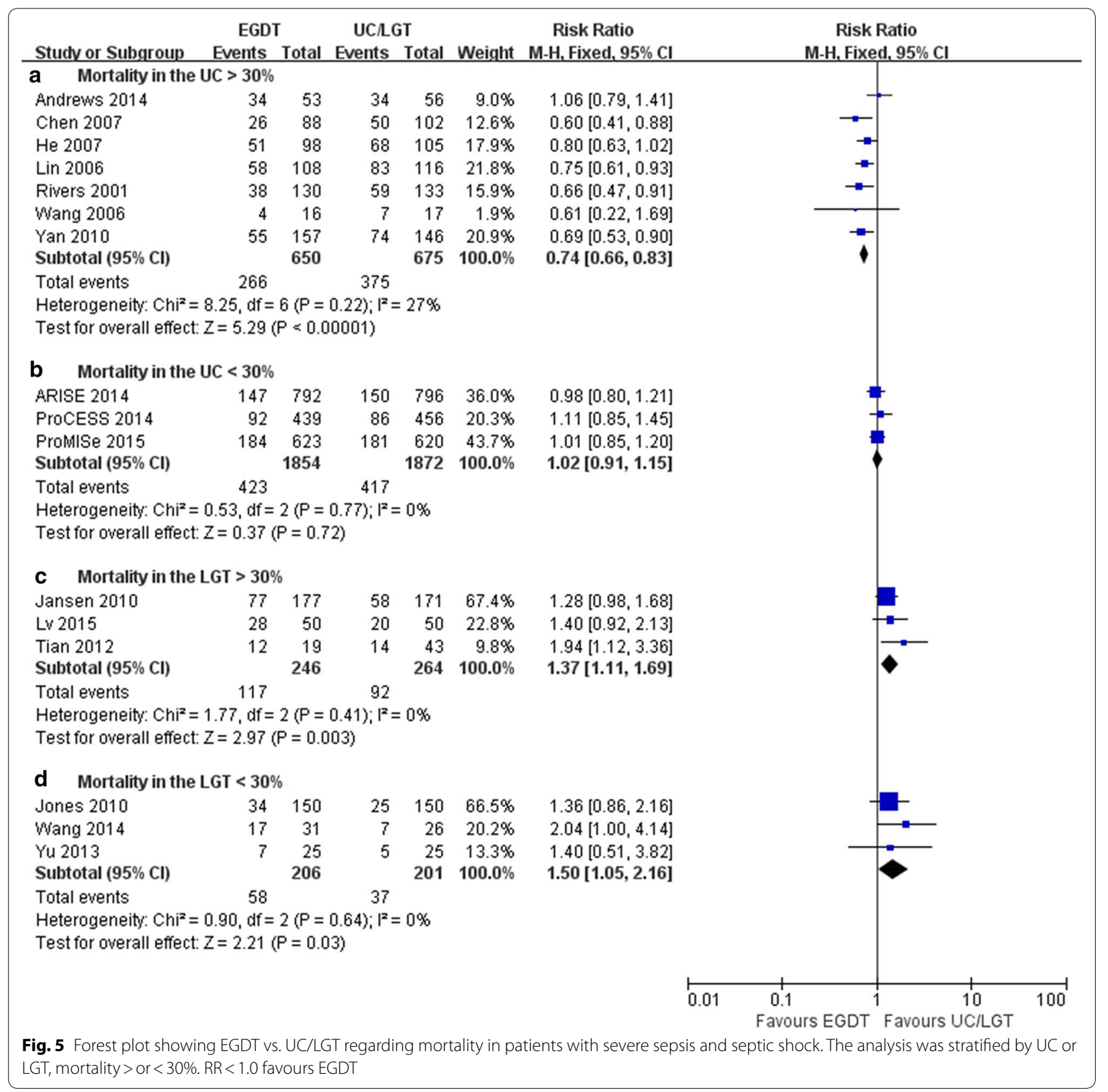

trials provided data comparing the secondary outcomes of EGDT and UC. As shown in Fig. 6, the pooled results showed that within the first $6 \mathrm{~h}$, patients in the EGDT groups received greater total volumes of red cell transfusions (6 trials; $\mathrm{RR}=1.83,95 \% \mathrm{CI} 1.29-2.60, P=0.0007$; $\left.I^{2}=78 \%, P=0.0003\right)[4,6-8,28,31]$, more dobutamine (6 trials; RR $=3.49,95 \%$ CI $1.58-7.70, P=0.002 ; I^{2}=84 \%$, $P<0.0001)[4,6-8,28,31]$, and more vasopressor infusions (5 trials; $\mathrm{RR}=1.15,95 \%$ CI $1.09-1.21, P<0.0001$; $\left.I^{2}=0 \%, P=0.45\right)[4,6-8,31]$ than UC patients, but no difference in the mechanical ventilation rate was found between the two groups ( 5 trials; $\mathrm{RR}=1.06,95 \% \mathrm{CI}$ $\left.0.97-1.16, P=0.18 ; I^{2}=0 \%, P=0.73\right)[4,6-8,32]$. The groups did not differ significantly regarding APACHE II scores $>20$ and $<20(\mathrm{RR}=0.84,95 \%$ CI $0.56-1.26$, $P=0.39 ; I^{2}=71 \%, P=0.03$; and $\mathrm{RR}=0.89$, 95\% CI $0.70-$ $1.13, P=0.35 ; I^{2}=68 \%, P=0.05$, respectively).

As shown in Fig. 7, a total of 3 trials [13-15] provided information on the comparisons of EGDT and LGT regarding secondary outcomes. Red cell transfusions, vasopressor infusions, dobutamine use, and the mechanical ventilation rate did not significantly differ between 


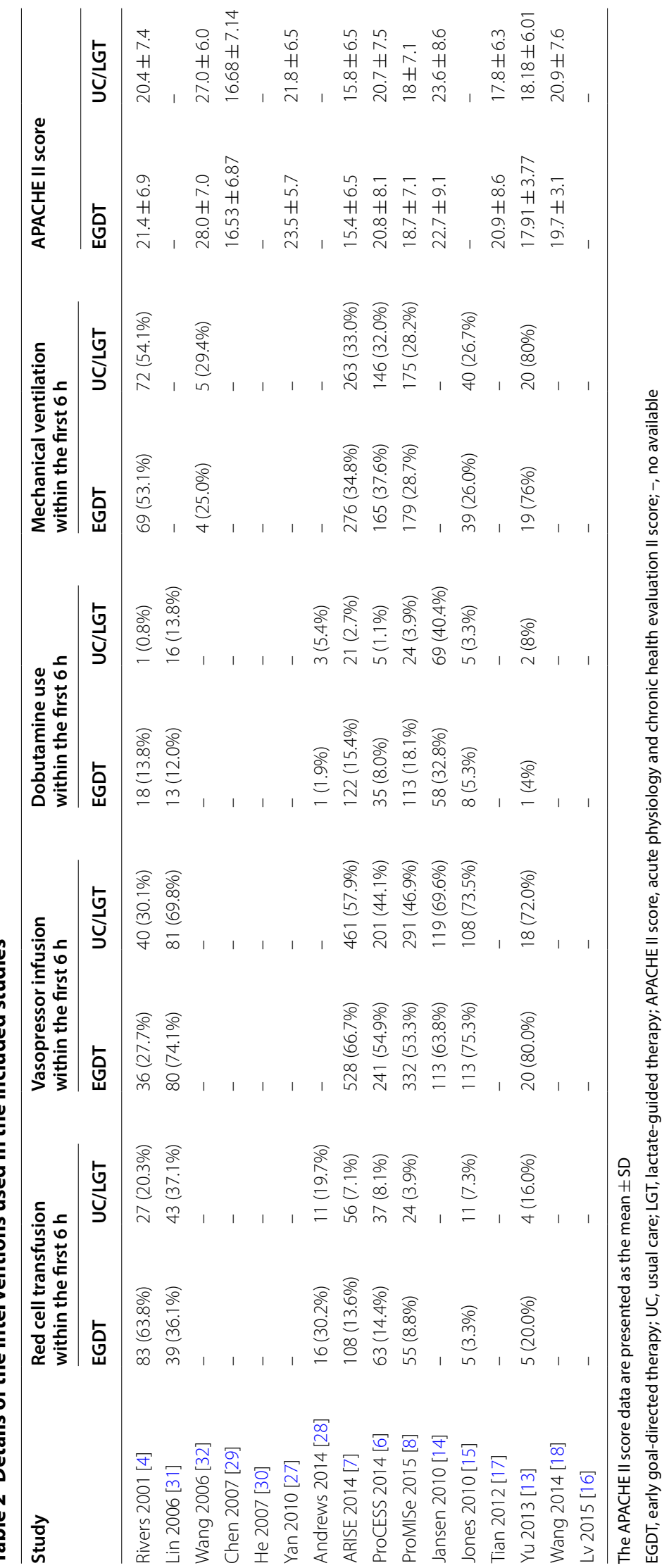


(See figure on next page.)

Fig. 6 Forest plot showing EGDTvs. UC regarding red cell transfusions, dobutamine use, vasopressor infusion, and mechanical ventilation within the first $6 \mathrm{~h}$ and APACHE II score in patients with severe sepsis and septic shock. The severity of illness was reported in each study by the APACHE II score, and the data were presented as the mean \pm SD. The distinction between higher and lower severity of illness was determined according to the mean APACHE II score in each study. APACHE I| scores $>$ or $<20$ were reported in 3 trials. RR $<1.0$ favours EGDT

the EGDT and LGT groups in the fixed-effects model $\left(\mathrm{RR}=0.67,95 \%\right.$ CI $0.31-1.43, P=0.30 ; I^{2}=37 \%, P=0.21$; $\mathrm{RR}=0.99,95 \%$ CI $0.90-1.09, P=0.80 ; I^{2}=8 \%, P=0.34$; $\mathrm{RR}=0.86$, 95\% CI $0.65-1.12, P=0.25 ; I^{2}=0 \%, P=0.45$; and $\mathrm{RR}=0.97,95 \%$ CI $0.74-1.27, P=0.81 ; I^{2}=0 \%$, $P=0.90$, respectively).

\section{Discussion \\ Main findings \\ EGDT vs. UC}

This meta-analysis showed that EGDT resulted in a $15 \%$ reduction in all-cause mortality compared with UC in adult patients with severe sepsis and septic shock, and this difference was particularly evident in the subgroup of UC patients with a mortality rate $>30 \%$. The results also showed that EGDT had a significant mortality benefit prior to publication of the SSC2012.

Given these results, the efficacy of EGDT remains unclear, as the ProCESS, ARISE, and ProMISe trials [68] compared EGDT with UC and failed to show a mortality reduction. However, the results of these 3 trials [6-8] are highly controversial.

In this study, patients assigned to the EGDT group received more red cell transfusions, dobutamine and vasopressor infusions within the first $6 \mathrm{~h}$ than those assigned to UC. However, EGDT did not significantly differ from UC in the use of mechanical ventilation.

\section{EGDT vs. LGT}

We found that EGDT resulted in higher all-cause mortality compared with LGT regardless of whether the subgroup receiving LGT had a mortality rate $>$ or $<30 \%$. In otherwords, another strength of our findings was that LGT was found to decrease mortality compared with EGDT. Furthermore, use of red cell transfusions, dobutamine, vasopressor infusions, and mechanical ventilation did not differ between EGDT and LGT. Jansen et al. [14] reported that the hospital mortality of EGDT in ICU was $43.5 \%$, and which of LGT was $33.9 \%$. This study suggests that increased blood lactate levels have been associated with significant morbidity and mortality in ICU patients, and initial treatment aimed at reducing lactate levels has clinical benefit. It also indicated in patients with hyperlactatemia on ICU admission, lactate monitoring followed by targeted treatment significantly reduced ICU length of stay. However, monitoring itself cannot improve outcome; therefore, the therapeutic plan associated with the monitoring is equally important. The main differences in therapy between the two groups in the treatment period were the administration of more fluids and the increased use of vasodilators in patients assigned to the lactate group. In addition, Wang et al. [18] and Tian et al. [17] reported that LGT had a more lower mortality than EGDT in sepsis. However, we need multicentre, large sample clinical trials and animal studies to explore the mechanism of LGT in the treatment of sepsis.

\section{Comparison with other studies}

After the ProCESS, ARISE, and ProMISe trials [6-8] failed to show that EGDT decreased mortality in severe sepsis and septic shock, some studies [33-35] updated their meta-analyses to provide the latest and most convincing evidence regarding EGDT in sepsis. Similar to the findings of previous meta-analyses [33-35], our study found that EGDT was associated with lower mortality than UC, especially in more severe patients. We aimed to explain this difference in results by considering several factors. First, the mortality of the three wellknown trials [6-8] was reported for UC patients with a mortality $<30 \%$ (Fig. 5). Consistent with the findings of our meta-analysis, EGDT has shown significant mortality benefits when the mortality of UC is $>30 \%$. Second, we found that patients assigned to EGDT received more red cell transfusions, dobutamine and vasopressor infusions than patients in the UC groups. The SSC 2016 [9] recommended that red cell transfusion occur only when the haemoglobin concentration decreases to $<7.0 \mathrm{~g} / \mathrm{dl}$ in adults in the absence of extenuating circumstances, such as myocardial ischemia, severe hypoxemia, or acute haemorrhage (strong recommendation, high quality of evidence). In addition, EGDT patients received longer mechanical ventilation, but this difference was not significant. In conclusion, EGDT may lead to more positive outcomes than UC. The SSC 2016 also stated that early effective fluid resuscitation is critical to stabilization of sepsis-induced tissue hypoperfusion or septic shock [9]. Third, Levy [36] raised the question of how 'usual' care and 'real-world' care are defined. The treatment provided with UC has increasingly trended towards that proposed by Rivers [4]. In Fig. 3, the RRs gradually neared 1 when arranging the trials in chronological order. This finding indicates that UC treatment gradually changed over time. 


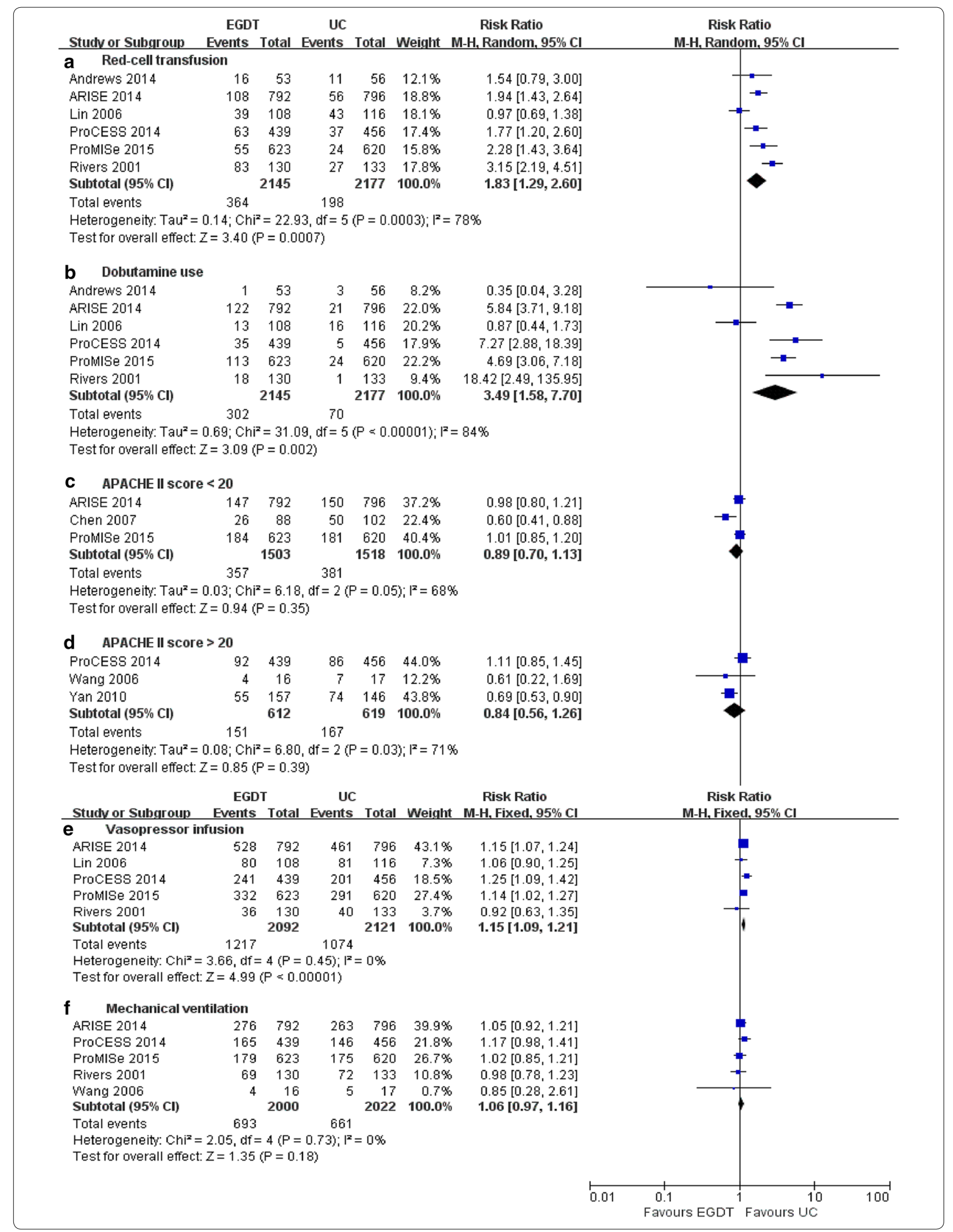




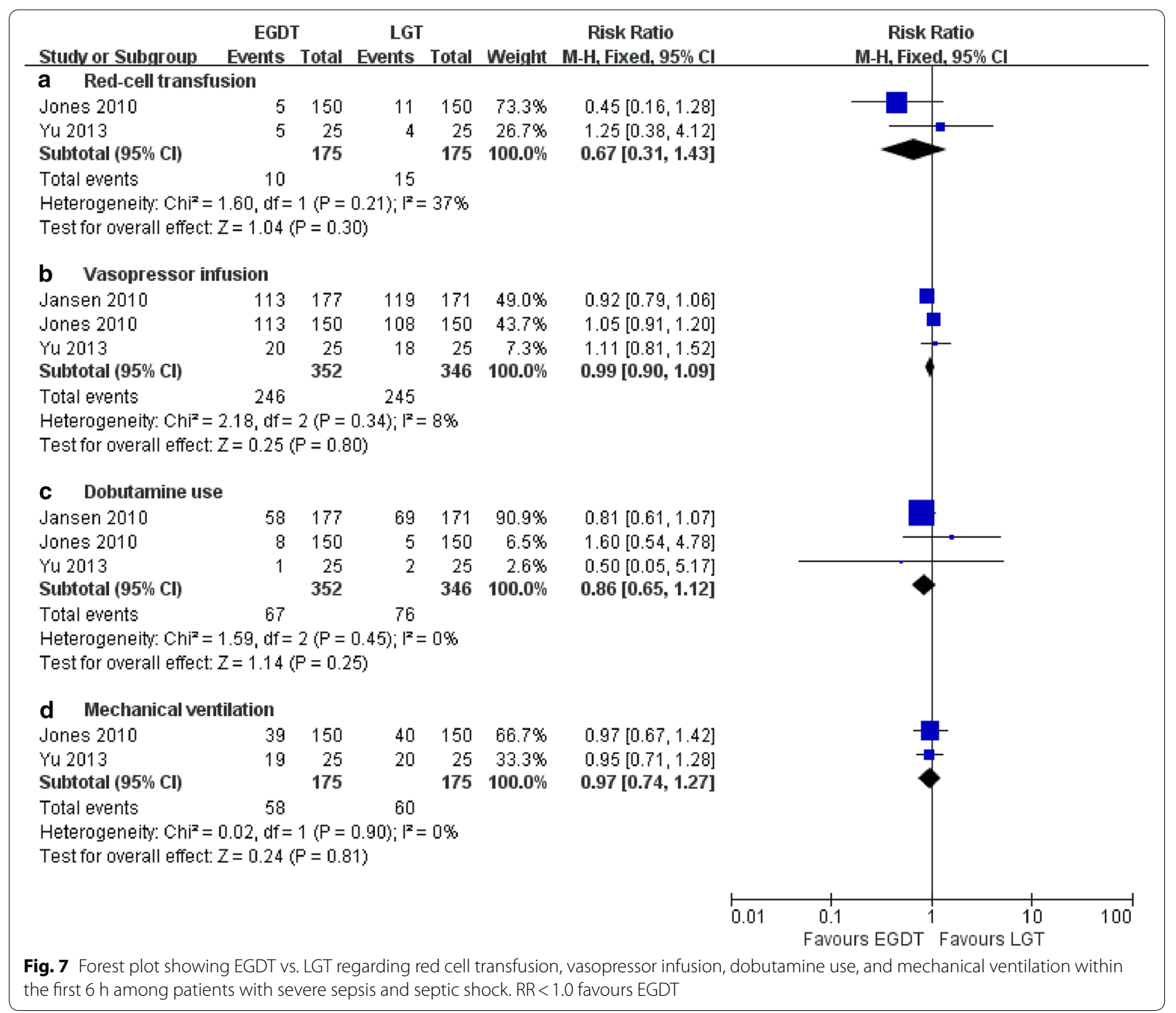

How do our study results compare with those of previous meta-analyses? Gu et al. [34] analysed only four randomized trials; thus, their results may be false-positive or may not be as robust or reliable. Lu et al. [35] did not conduct detailed or comprehensive subgroup analyses to assess the differences between EGDT and LGT and included only 13 studies. Finally, another study by $\mathrm{Gu}$ et al. [33] included studies from a different time period.

In sepsis, a more controversial issue is the index used to reflect tissue oxygen delivery. The SSC 2012 recommended using $\mathrm{Scvo}_{2}$ to assess the balance between tissue oxygen delivery and consumption [37]. However, there are still some challenges to identifying patients with sepsis, and the largest roadblocks to overcome in implementing $\mathrm{Scvo}_{2}$ include time, expertise, adherence, and need for specialized equipment [38]. Currently, studies have found that blood lactate concentration could be used to reflect the restoration of oxygen delivery in resuscitation and have confirmed that lactate monitoring is useful [39]. However, the optimal measurement choice in different situations remains unclear. The ProCESS, ARISE, and ProMISe trials were unable to recommend using $\mathrm{Scvo}_{2}$ from their findings. In addition, the SSC2016 recommended viewing these patients as experiencing a medical emergency requiring urgent assessment and treatment and suggested guiding resuscitation by normalizing lactate inpatients with elevated lactate levels, as these levels may serve as a marker of tissue hypoperfusion [9]. As LGT is non-invasive, when the same monitoring effort is expended, we can recommend LGT as the better option. 


\section{Strengths and limitations}

This meta-analysis included 10 RCTs that systematically compared EGDT with UC in 5051 patients, and the results showed a $15 \%$ reduction in all-cause mortality in adult patients with severe sepsis and septic shock. Another strength of this study was the finding that LGT may have a greater mortality benefit than EGDT.

Our study has several limitations. First, seven trials [17, $18,25,27,29,30,32]$ had an unclear risk of bias. Second, the effect of region-specific variations in clinical practice could not be assessed in our meta-analysis.

\section{Conclusions}

Our meta-analysis showed that a 6-h EGDT protocol for patients with severe sepsis or septic shock resulted in lower mortality than UC, especially in severely ill patients. The most likely reason for this finding is that patients assigned to EGDT received more red cell transfusions, dobutamine, and vasopressor infusions within the first $6 \mathrm{~h}$. Compared with EGDT, LGT was associated with lower mortality, despite the lack of differences in the use of red cell transfusions, dobutamine, vasopressor infusion, and mechanical ventilation. However, in the future, we look forward to an updated meta-analysis including more high-quality RCTs mainly related to LGT versus EGDT and taking into account red cell transfusions, vasopressor infusions, dobutamine use, and the use of mechanical ventilation within the first $6 \mathrm{~h}$, which may provide more precise guidance for clinicians in the treatment of sepsis.

\section{Additional file}

Additional file 1. PRISMA 2009 checklist.

\section{Abbreviations}

EGDT: early goal-directed therapy; LGT: lactate-guided therapy; UC: usual care; $\mathrm{RCT}$ : randomized controlled trials; ED: emergency department; $\mathrm{SCVO}_{2}$ : systemic central venous oxygen saturation; SSC: Surviving Sepsis Campaign; APACHE II: Acute Physiology and Chronic Health Evaluation II; PRISMA: preferred reporting items for systematic reviews and meta-analyses; RR: relative risk; Cl: confidence interval; M-H: Mantel-Haenszel.

\section{Authors' contributions}

XFD and ZYY wrote the manuscript paper. TWS and XZ designed the study. $X F D, Z Y Y, Z T X, L F L, B Y, L N G$, and LXW collected data, performed some analysis. All authors read and approved the final manuscript.

\footnotetext{
Author details

${ }^{1}$ General ICU, The First Affiliated Hospital of Zhengzhou University, Zhengzhou 450052, China. ${ }^{2}$ Henan Key Laboratory of Critical Care Medicine, Zhengzhou 450052, China. ${ }^{3}$ Department of Pharmacy, The First Affiliated Hospital of Zhengzhou University, Zhengzhou 450052, China. ${ }^{4}$ Biotherapy Center, The First Affiliated Hospital of Zhengzhou University, Zhengzhou 450052, China. ${ }^{5}$ Department of Neurology, The First Affiliated Hospital of Zhengzhou University, Zhengzhou 450052, China. ${ }^{6}$ School of Biomedical Sciences, Charles
}

Sturt University, Wagga 2650, Australia. ${ }^{7}$ Department of Critical Care Medicine, Peking University Third Hospital, Beijing 100191, China.

\section{Acknowledgements \\ None.}

Competing interests

The authors declare that they have no competing interests.

Availability of data and materials

All data generated or analysed during this study are included in this published article [and its additional file].

\section{Consent for publication}

Not applicable.

\section{Ethics approval and consent to participate}

Not applicable.

\section{Funding}

This work was supported by the Grants from the National Natural Science Foundation of China (No. 81370364), the Science and Technology Innovation Team at the Universities of Henan province (No. 16IRTSTHN021), the "51282" Project Leaders of Scientific and Technological Innovative Talents from Health and Family Planning Commission in Henan Province (2016-32).

\section{Publisher's Note}

Springer Nature remains neutral with regard to jurisdictional claims in published maps and institutional affiliations.

Received: 27 June 2018 Accepted: 20 November 2018

Published online: 29 November 2018

\section{References}

1. Torio CM, Andrews RM. National inpatient hospital costs: the most expensive conditions by payer 2007: statistical Brief\#160[A]. Healthcare Cost and Utilization Project (HCUP) Statistical Briefs. Rockville: Agency for Healthcare Research and Quality; 2006.

2. Vincent JL, Marshall JC, Namendys-Silva SA, et al. Assessment of the worldwide burden of critical illness: the intensive care over nations (ICON) audit. Lancet Respir Med. 2014;2(5):380-6.

3. Fleischmann C, Scherag A, Adhikari NK, et al. Assessment of global incidence and mortality of hospital-treated sepsis. Current estimates and limitations. Am J Respir Crit Care Med. 2016;193(3):259-72.

4. Rivers E, Nguyen B, Havstad S, et al. Early goal-directed therapy in the treatment of severe sepsis and septic shock. N Engl J Med. 2001;345(19):1368-77.

5. Nguyen $\mathrm{HB}$, Jaehne AK, Jayaprakash N, et al. Early goal-directed therapy in severe sepsis and septic shock: insights and comparisons to ProCESS, ProMISe, and ARISE. Crit Care. 2016;20(1):160.

6. Yealy DM, Kellum JA, Huang DT, et al. A randomized trial of protocolbased care for early septic shock. N Engl J Med. 2014;370(18):1683-93.

7. Peake SL, Delaney A, Bailey M, et al. Goal-directed resuscitation for patients with early septic shock. N Engl J Med. 2014;371(16):1496-506.

8. Mouncey PR, Osborn TM, Power GS, et al. Trial of early, goal-directed resuscitation for septic shock. N Engl J Med. 2015;372(14):1301-11.

9. Rhodes A, Evans LE, Alhazzani W, et al. Surviving sepsis campaign: international guidelines for management of sepsis and septic shock: 2016. Intensive Care Med. 2017:43(3):304-77.

10. Casserly B, Phillips GS, Schorr C, et al. Lactate measurements in sepsisinduced tissue hypoperfusion: results from the surviving sepsis campaign database. Crit Care Med. 2015;43(3):567-73.

11. Nguyen HB, Rivers EP, Knoblich BP, et al. Early lactate clearance is associated with improved outcome in severe sepsis and septic shock. Crit Care Med. 2004;32(8):1637-42.

12. Arnold RC, Shapiro NI, Jones $A E$, et al. Multicenter study of early lactate clearance as a determinant of survival in patients with presumed sepsis. Shock. 2009;32(1):35-9. 
13. Yu B, Tian HY, Hu ZJ, et al. Comparison of the effect of fluid resuscitation as guided either by lactate clearance rate or by central venous oxygen saturation in patients with sepsis. Zhonghua Wei Zhong Bing Ji Jiu Yi Xue. 2013;25(10):578-83.

14. Jansen TC, van Bommel J, Schoonderbeek FJ, et al. Early lactate-guided therapy in intensive care unit patients: a multicenter, open-label, randomized controlled trial. Am J Respir Crit Care Med. 2010;182(6):752-61.

15. Jones AE, Shapiro NI, Trzeciak S, et al. Lactate clearance vs central venous oxygen saturation as goals of early sepsis therapy: a randomized clinical trial. JAMA. 2010;303(8):739-46.

16. Lyu X, Xu Q, Cai G, et al. Efficacies of fluid resuscitation as guided by lactate clearance rate and central venous oxygen saturation in patients with septic shock. Zhonghua Yi Xue Za Zhi. 2015;95(7):496-500.

17. Tian HH, Han SS, LV CJ, et al. The effect of early goal lactate clearance rate on the outcome of septic shock patients with severe pneumonia. Zhongguo Wei Zhong Bing Ji Jiu Yi Xue. 2012;24(1):42-5.

18. Wang $T, X i a Y, H a o D$, et al. The significance of lactic acid in early diagnosis and goal-directed therapy of septic shock patients. Zhonghua Wei Zhong Bing Ji Jiu Yi Xue. 2014;26(1):51-5.

19. Jones $A E$, Shapiro NI, Roshon M. Implementing early goal-directed therapy in the emergency setting: the challenges and experiences of translating research innovations into clinical reality in academic and community settings. Acad Emerg Med. 2007;14(11):1072-8.

20. Angus DC, Barnato AE, Bell D, et al. A systematic review and meta-analysis of early goal-directed therapy for septic shock: the ARISE, ProCESS and ProMISe Investigators. Intensive Care Med. 2015;41(9):1549-60.

21. Jiang LB, Zhang M, Jiang SY, et al. Early goal-directed resuscitation for patients with severe sepsis and septic shock: a meta-analysis and trial sequential analysis. Scand J Trauma Resusc Emerg Med. 2016;24:23.

22. Lu J, Wang X, Chen Q, et al. The effect of early goal-directed therapy on mortality in patients with severe sepsis and septic shock: a meta-analysis. J Surg Res. 2016;202(2):389-97.

23. Simpson SQ, Gaines M, Hussein Y, et al. Early goal-directed therapy for severe sepsis and septic shock: a living systematic review. J Crit Care. 2016:36:43-8.

24. Moher D, Liberati A, Tetzlaff J, et al. Preferred reporting items for systematic reviews and meta-analyses: the PRISMA statement. Int J Surg. 2010;8(5):336-41.

25. Dellinger RP, Levy MM, Rhodes A, et al. Surviving Sepsis Campaign: international guidelines for management of severe sepsis and septic shock, 2012. Intensive Care Med. 2013;39(2):165-228.
26. Higgins JP, Thompson SG, Deeks JJ, et al. Measuring inconsistency in meta-analyses. BMJ. 2003;327(7414):557-60.

27. Early Goal-Directed Therapy Collaborative Group of Zhejiang Province. The effect of early goal-directed therapy on treatment of critical patients with severe sepsis/septic shock: a multi-center, prospective, randomized, controlled study. Zhongguo Wei Zhong Bing Ji Jiu Yi Xue. 2010;22(6):331-4.

28. Andrews B, Muchemwa L, Kelly P, et al. Simplified severe sepsis protocol: a randomized controlled trial of modified early goal-directed therapy in Zambia. Crit Care Med. 2014;42(11):2315-24.

29. Chen $\mathrm{ZQ}$, Jin $\mathrm{YH}$, Chen $\mathrm{H}$, et al. Early goal-directed therapy lowers the incidence, severity and mortality of multiple organ dysfunction syndrome. Nan Fang Yi Ke Da Xue Xue Bao. 2007;27(12):1892-5.

30. He ZY, Gao Y, Wang XR, et al. Clinical evaluation of execution of early goal directed therapy in septic shock. Zhongguo Wei Zhong Bing Ji Jiu Yi Xue. 2007;19(1):14-6.

31. Lin SM, Huang CD, Lin HC, et al. A modified goal-directed protocol improves clinical outcomes in intensive care unit patients with septic shock: a randomized controlled trial. Shock. 2006;26(6):551-7.

32. Wang XZ, Lu CJ, Gao FQ, et al. Efficacy of goal-directed therapy in the treatment of septic shock. Zhongguo Wei Zhong Bing Ji Jiu Yi Xue. 2006;18(11):661-4.

33. Gu WJ, Wang F, Bakker J, et al. The effect of goal-directed therapy on mortality in patients with sepsis-earlier is better: a meta-analysis of randomized controlled trials. Crit Care. 2014;18(5):570.

34. Gu WJ, Zhang Z, Bakker J. Early lactate clearance-guided therapy in patients with sepsis: a meta-analysis with trial sequential analysis of randomized controlled trials. Intensive Care Med. 2015;41(10):1862-3.

35. Lu Y, Zhang $H$, Teng $F$, et al. Early goal-directed therapy in severe sepsis and septic shock: a meta-analysis and trial sequential analysis of randomized controlled trials. J Intensive Care Med. 2016:33:296-309.

36. Levy MM. Early goal-directed therapy: what do we do now? Crit Care. 2014;18(6):705

37. Dellinger RP, Levy MM, Rhodes A, et al. Surviving sepsis campaign: international guidelines for management of severe sepsis and septic shock: 2012. Crit Care Med. 2013;41(2):580-637.

38. Carlbom DJ, Rubenfeld GD. Barriers to implementing protocol-based sepsis resuscitation in the emergency department-results of a national survey. Crit Care Med. 2007;35(11):2525-32.

39. Bakker J, Nijsten MW, Jansen TC. Clinical use of lactate monitoring in critically ill patients. Ann Intensive Care. 2013;3(1):12.
Ready to submit your research? Choose BMC and benefit from:

- fast, convenient online submission

- thorough peer review by experienced researchers in your field

- rapid publication on acceptance

- support for research data, including large and complex data types

- gold Open Access which fosters wider collaboration and increased citations

- maximum visibility for your research: over $100 \mathrm{M}$ website views per year

At $\mathrm{BMC}$, research is always in progress.

Learn more biomedcentral.com/submissions 